Transport and Communications Science Journal

\title{
A PROPOSAL OF METHODOLOGY TO ANALYZE PLATOON DISPERSION AT SIGNALIZED CROSSWALK CONSIDERING PEDESTRIAN DEMAND AND CROSSWALK LENGTH
}

\author{
Chu Tien Dung ${ }^{*}$
}

${ }^{1}$ Vietnam - Japan Research and Development Center (Vijard), University of Transport and Communications, No 3 Cau Giay Street, Hanoi, Vietnam.

\author{
ARTICLE INFO \\ TYPE: Research Article \\ Received: 27/09/2019 \\ Revised: 22/11/2019 \\ Accepted: 30/11/2019 \\ Published online: 31/01/2020 \\ https://doi.org/10.25073/tcsj.71.1.1 \\ *Corresponding author \\ Email: dungchu@utc.edu.vn
}

\begin{abstract}
Understanding pedestrian platoon dispersion at signalized crosswalk is essential not only for improving pedestrian safety but also for estimating pedestrian crossing time requirement. This paper proposed platoon dispersion ratio (PDR) as an index to analyse the effects pedestrian demand and crosswalk length upon pedestrian platoon dispersion at signalized crosswalk. The result shows that the heavier pedestrian demand usually corresponds to greater platoon dispersion. Regarding the influence of crosswalk length, it is revealed that the dispersion tends to increase as the crosswalk length increases.
\end{abstract}

Keywords: Pedestrian platoon, dispersion, dispersion ratio, pedestrian demand, crosswalk length

(C) 2020 University of Transport and Communications

\section{INTRODUCTION}

In the last few decades, researchers and planers have paid great attention to pedestrians in order to improve their comfort and safety, especially at signalized crosswalks. At signalized crosswalks, although pedestrians have prioritized right of way, the pedestrian fatalities due to traffic accident is still high. The threat to pedestrian safety mainly comes from the turning vehicles since in common phasing plans; they share the same phase with pedestrians. In European Union (EU), as of 2016, 5.320 pedestrians were killed in road accidents in the EU (excluding Lithuania and Slovakia), which is $21 \%$ of all road fatalities (see Fig.1) [1]. In Japan, as shown in Fig. 2 nearly one-third of the total traffic accident 
Transport and Communications Science Journal, Vol. 71, Issue 1 (01/2020), 1-10

fatalities are pedestrians [2]. Understanding both pedestrian and vehicle behavior is very important for improving the safety performance of signalized intersection.

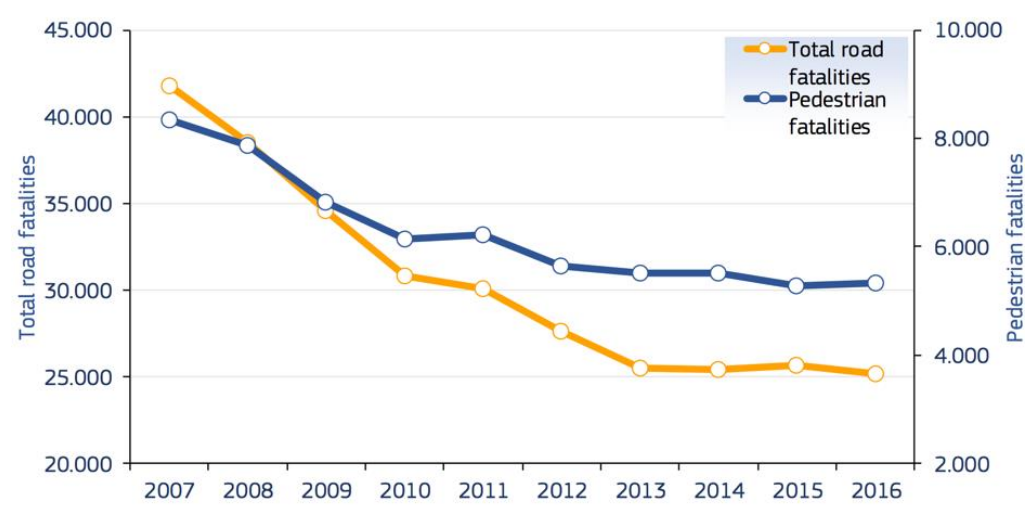

Figure 1. Pedestrian fatalities by total fatalities of all road users (Source: CARE database, data available in May 2018).

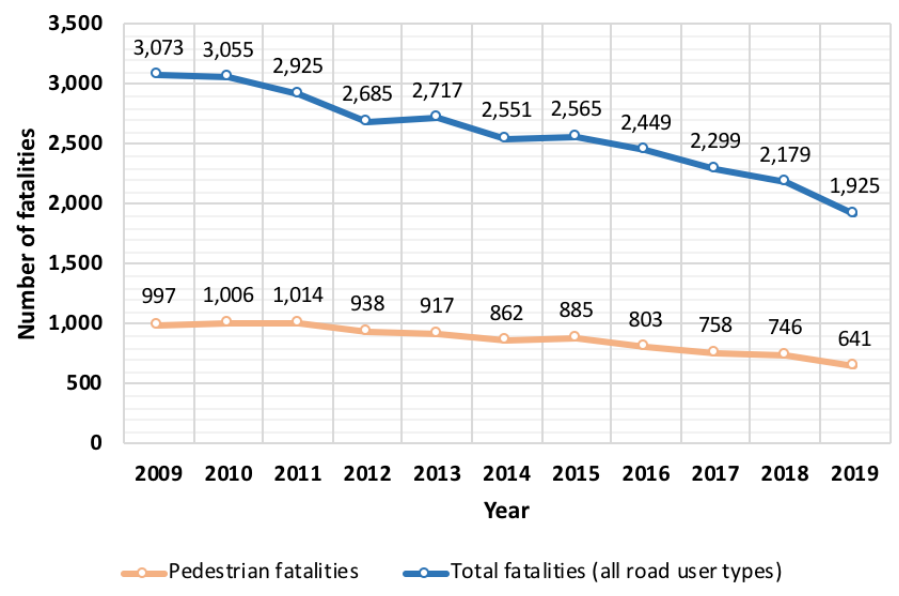

Figure 2. Pedestrian fatalities by total fatalities of all road users (source: Japan National Police Agency, 2019).

In reality, road users behave by anticipating other user's behavior. When pedestrian platoon moves to the downstream of the crosswalk and disperses, the gap may appear for the turning vehicles. The question is how pedestrian platoon disperses in different pedestrian demand and crosswalk length? Understanding this deformation in pedestrian platoon will be essential for predicting the gaps as well as accessing the safety of signalized intersection. In another aspect, how pedestrian platoon disperses as the platoon moves to the downstream of the crosswalk is essential for estimating pedestrian crossing time requirement at signalized crosswalks. However, most of the existing manuals and guidelines (e.g. U.S Highway Capacity Manual - HCM 2010 [3], Japanese Manual on Traffic Signal Control - 2006 [4]) for the design and operation of signalized intersections estimate the pedestrian clearance time depending on crosswalk length and constant speed without considering the effects of platoon dispersion.

Despite its importance, the studies regarding pedestrian platoon dispersion have not been properly considered. Although, many models have been established related to vehicle dispersion, they cannot be applied to pedestrian platoons. This is because pedestrian platoon dispersion is quite different from that for vehicle platoon. Compared to vehicles that follow 
each other on dedicated lanes, pedestrians can adjust their speed more freely, much less constrained by a platoon than vehicles to its platoon.

Thus, the aim of this study is to investigate the influencing factors in the dispersion of pedestrian platoon at signalized crosswalks in order to improve pedestrian safety as well as the operation and design of signalized crosswalks. This paper concentrates upon the influences of pedestrian demand and crosswalk length on pedestrian platoon dispersion.

This paper is organized as follows: after the introduction and literature review, data collection and processing are explained. This is followed by analysis methodology. Then a comprehensive discussion on the effects of pedestrian demand and crosswalk length on pedestrian platoon dispersion are conducted and discussed. Finally, the paper ends up with summary of the results and future works.

\section{LITERATURE REVIEW}

Herein, two basic concepts are introduced from the literature regarding pedestrian platoon. The U.S Highway Capacity Manual (HCM 2010) [3] defines pedestrian platoon as a group of pedestrian traveling together either voluntarily or involuntarily, because of signal control, road geometry or other factors. In a study of Arasan and Kashani [5], platoon dispersion is defined as the phenomenon, in which pedestrians released from, for example, a waiting area, will get segregated, as they move to the downstream of the crosswalk.

Virkler [6] analysed the potential benefits of reducing delay through consideration of pedestrian dispersion. He concluded that if pedestrians can select their own speeds along a sidewalk, platoon dispersion will naturally occur. Moreover, when pedestrian flow reaches the end of the crosswalk, dispersion occurred and it would continue to disperse as pedestrian flow moves further along the sidewalk. However, the dispersion formulation and its influencing factors are not clear. In another studies, Virkler [7] proposed a model for estimating crossing time at scramble crossing and two-way crosswalk (Eq. 1). The model is then adopted by HCM 2010. He found that the platoon dispersion has significant effects on estimating required pedestrian crossing time. Therefore, in his proposed model, the effects of platoon dispersion were considered by simply adding amount of time for each pedestrian into platoon discharge time (the last part).

$$
\begin{array}{ll}
T=I+\frac{L}{S p}+0.27 N_{p e d} & \text { for } w \leq 3 m \\
T=I+\frac{L}{S p}+0.81 \frac{N_{p e d}}{w} & \text { for } w>3 m
\end{array}
$$

Where:

$T$ is total time required for all the crossing process,

$I$ is initial start-up lost time,

$L$ is crosswalk length $(\mathrm{m})$,

$S_{p}$ is walking speed $(\mathrm{m} / \mathrm{sec})$,

$N_{\text {ped }}$ is number of pedestrians crossing during an interval $\mathrm{p}$ from one side of the crosswalk,

And $w$ is crosswalk width (m).

Although the model considers the effects of platoon dispersion, simply adding time headway for each pedestrian might be not enough. Because the dispersion is not influenced 
by only pedestrian demand but also other factors such as bi-directional effect, pedestrian origin-destination, crosswalk length, etc. The Japanese Manual on Traffic Signal Control (2006) [4] proposes a formula to estimate required crossing time as presented in Equation 2. However, the proposed model does not consider the effects of platoon dispersion.

$$
T=\frac{L}{S p}+\frac{N_{p e d}}{Q_{d} w}
$$

Where $Q_{d}$ is discharge rate (ped/m.sec).

Recently, Forde and Daniel [8] uses observed data to evaluate the performance of the platoon dispersion model of HCM 2010. The model was evaluated under influences and without influences of traffic conditions including pedestrian and vehicular interactions on urban collector street segments and the presence of trucks within the traffic stream on principal urban arterial segments. It is concluded that the HCM 2010 platoon dispersion model performs relatively well in predicting platoon arrival profiles on urban street segments without influences of traffic conditions. However, under influences of pedestrian and truck, the model's performance is limited. Wael et al [9] investigates the rationality and effectiveness of designing signal coordination for pedestrians considering relationship between pedestrian platoon dispersion and the signal cycle length. Their results showed that the coordination for the major pedestrian flow led to a significant reduction in average delay (15\%).

\section{DATA COLLECTION AND PROCESSING}

For analyzing pedestrian platoon dispersion, video data was collected at three signalized crosswalks in Japan (see Fig. 3). The first and the second sites (Mizuho Kuyakusho and Sasashima) are located in Nagoya City while the third one (Tokyo International Forum) is located in Tokyo City, Japan. The observation times, the geometric and pedestrian characteristics of the study sites are presented in Table 1. It is important to note that the pedestrian flows at these crosswalks are assumed to be unidirectional since the opposite pedestrian demand is very low (10\% compared to subject pedestrian demand). Therefore, the movement of platoon is almost not influenced by the opposing pedestrian flows.

The trajectories of crossing pedestrians are extracted from video data by using the image processing system TrafficAnalyze [10]. The position and timing of each pedestrian was extracted every 1.0 seconds. The point where the feet of the pedestrian are touching the ground is the reference observation point. Notably, an advantage of the TrafficAnalyze is that it helps to collect the trajectory of each individual pedestrian. However, it has some disadvantages. For example, the accuracy of trajectory is dependent on quality of video resolution, angle of camera and so on.

Table 1. Surveyed site characteristics.

\begin{tabular}{lccc}
\hline Intersection name & Mizuho Kuyakusho & Sasashima & Tokyo Forum \\
\hline Crosswalk position & East leg & East leg & South leg \\
\hline Survey hour & $07: 00-09: 30$ & $07: 00-09: 30$ & $07: 30-10: 00$ \\
\hline Dimensions w $*$ L & $4(\mathrm{~m}) * 9(\mathrm{~m})$ & $5(\mathrm{~m}) * 15(\mathrm{~m})$ & $5(\mathrm{~m}) * 15(\mathrm{~m})$ \\
\hline Pedestrian type & Pupil & Business & Business \\
\hline Average demand & 682 peds/hr & 2025 peds $/ \mathrm{hr}$ & 660 peds $/ \mathrm{hr}$ \\
\hline
\end{tabular}



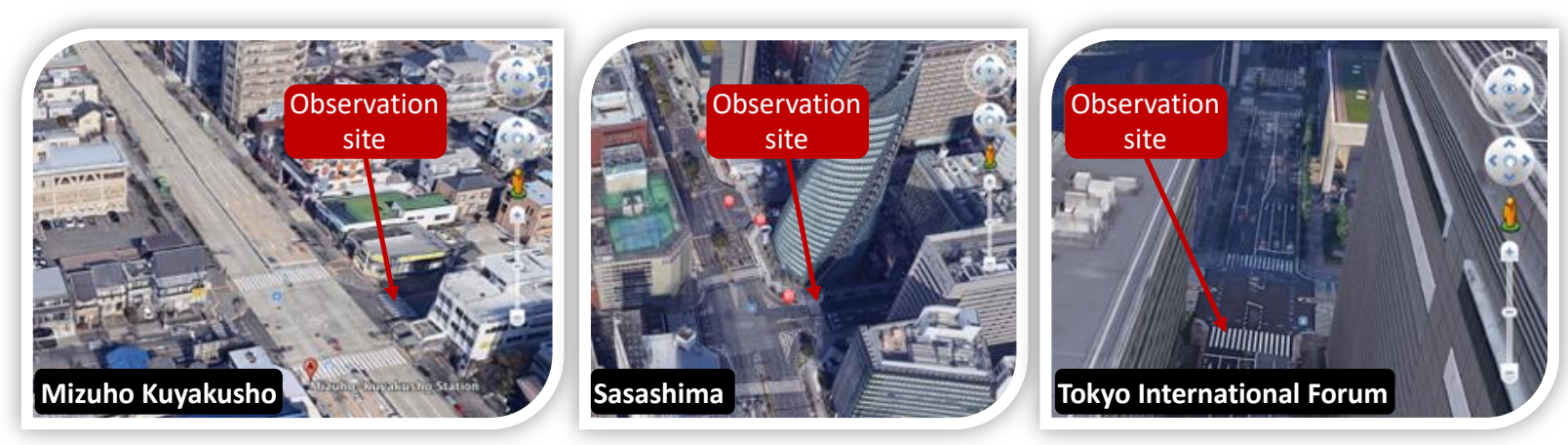

Figure 3. Observation sites.

\section{ANALYSIS METHODOLOGY}

Fig. 4 presents time-space diagram in order to compare the crossing time. In this study, it is assumed that, the upstream curb and downstream curb are defined based on the direction of pedestrians. From Fig. 4, it is obvious that there are several possible ways to measure pedestrian platoon dispersion.

- Comparing the average gap between successive pedestrian.

- Comparing the average flow rate at a downstream curb with the average flow rate at the upstream curb where the platoon was released.

- Comparing the relative time for the platoon to pass the upstream curb and downstream curb.

In this paper, considering the purpose of the analysis, the third method is utilized and platoon dispersion ratio (PDR) is proposed as a measure of platoon dispersion. In order to define PDR, the following assumptions are made:

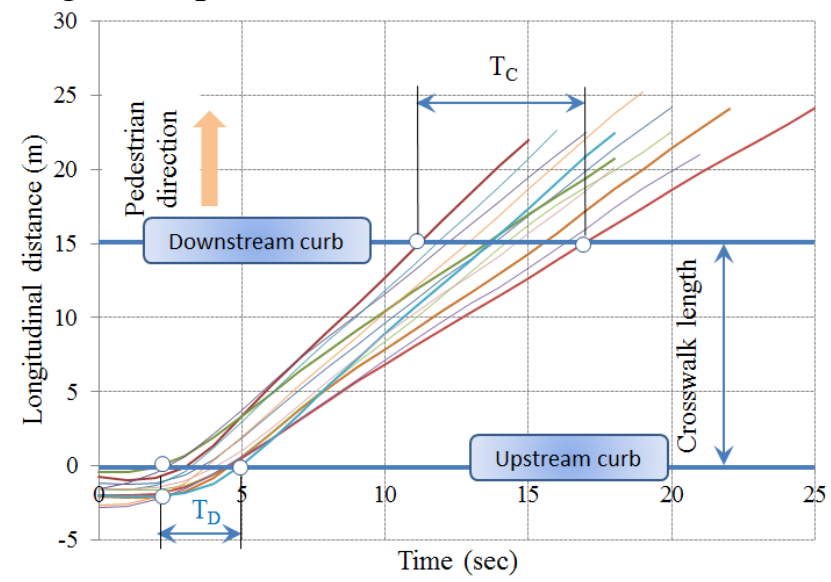

Figure 4. Pedestrian time-space diagram.

- A platoon is only considered when pedestrians are waiting before the onset of pedestrian green at waiting area and those are considered as one platoon only if the longitudinal distance between them less than 3 meters.

- When the platoon moves to the downstream of the crosswalk, the number of pedestrians in platoon keeps the same. It means that any possible division in the platoon is not considered. 
PDR is defined as shown in Eq. (3) and it is noted that the larger PDR, the greater platoon dispersion.

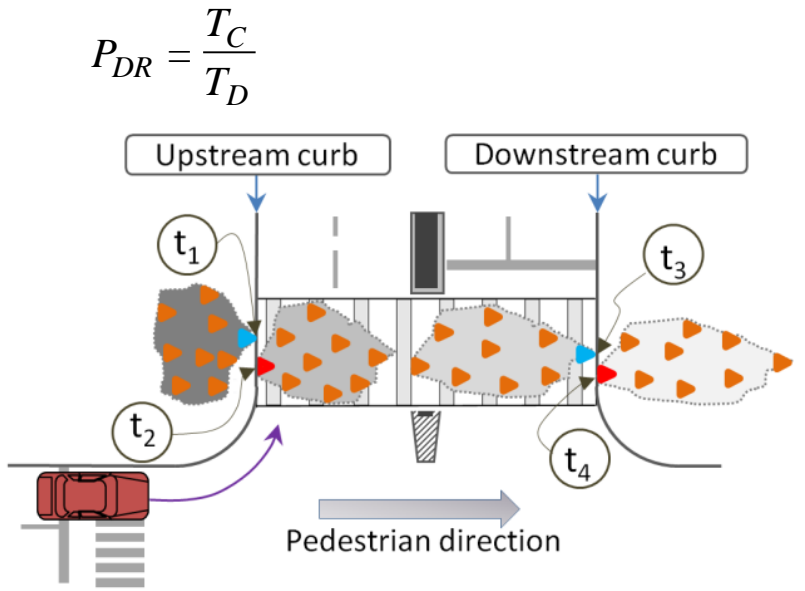

Figure 5. Definition of $t_{1}, t_{2}, t_{3}$ and $t_{4}$.

Where $T_{D}$ and $T_{C}$ are the necessary time for all of pedestrian within platoon to pass the upstream curb and downstream curb, respectively. Eq. 4 and Fig. 5 illustrates how $T_{C}$ and $T_{D}$ are calculated.

$$
\begin{aligned}
& T_{D}=t_{2}-t_{1} \\
& T_{C}=t_{4}-t_{3}
\end{aligned}
$$

In which:

$t_{1}$ and $t_{2}$ are the time at which the first and the last pedestrian in the platoon pass the upstream curb

$t_{3}$ and $t_{4}$ are the time at which the first and the last pedestrian in the platoon pass the downstream curb.

It should be noted that the first and the last pedestrian who pass the upstream and downstream curb might be different. PDR can be less than, equal or more than 1. If PDR is less than or equal to 1, it means that there is no dispersion at all. Such condition might happen when pedestrian demand is low to medium and they stand quite far from each other while waiting for the green signal. Therefore, pedestrians can get close to each other when platoon moves to the downstream of the crosswalk. If PDR is more than 1, the bigger PDR is, the greater dispersion occurs.

In another dimension, in order to evaluate the performance of existing methodology for the estimation of required pedestrian crossing time, the observed total crossing time and the total crossing time by HCM 2010 [3] and Japanese Manual on Traffic Signal Control 2006 [4] are estimated. The observed total crossing time is defined by the Equation 5 while the total crossing time by HCM (2010) and Japanese Manual are calculated assuming an average crossing speed of $1.2 \mathrm{~m} / \mathrm{sec}$ and $1.0 \mathrm{~m} / \mathrm{sec}$ respectively.

$$
\mathrm{T}=t_{4}-t_{1}
$$

Where $\mathrm{T}$ is observed total crossing time while $t_{1}$ and $t_{4}$ are explained above. 


\section{RESULTS AND DISCUSSION.}

\subsection{Comparison of total pedestrian platoon crossing time.}

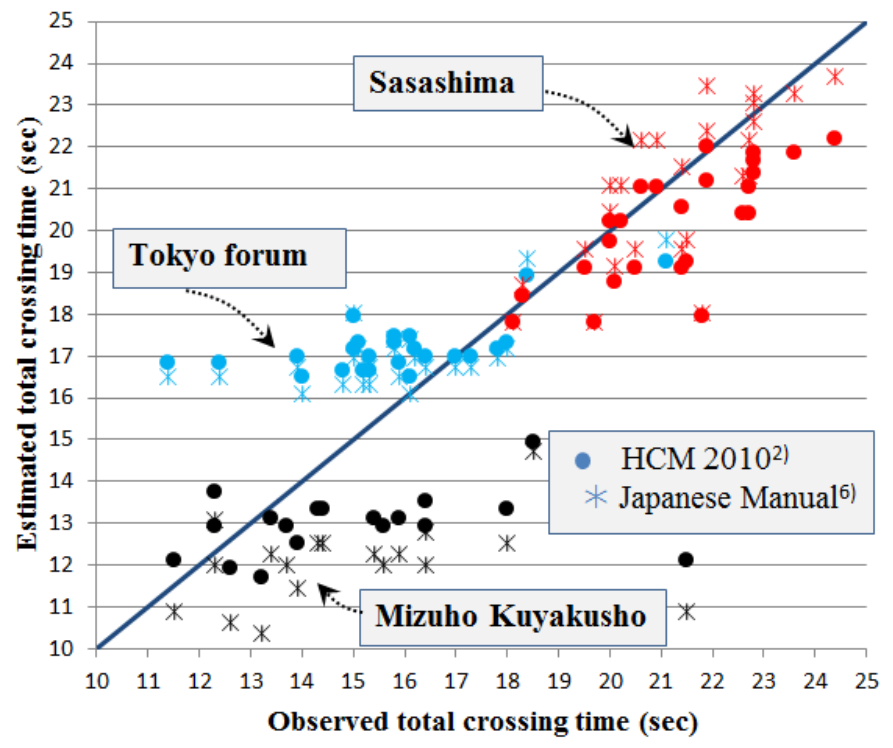

Figure 6. Total pedestrian platoon crossing time comparison.

The estimated total crossing times from HCM (2010), Japanese Manual (2006) and the observed total crossing time are compared as shown Fig. 6. From this figure, it can be seen that the total crossing time tends to overestimate at Mizuho Kuyakusho while underestimate at Tokyo forum crosswalk. It can be interpreted that at Mizuho Kuyakusho, the pedestrians are pupil so their speeds are slower compared to pedestrian speed at Tokyo forum crosswalk (business pedestrian). At Sasashima crosswalk with high pedestrian demand, the crossing time has a tendency of underestimation, especially HCM (2010) model. It might be that the effects of platoon dispersion are not satisfactorily considered in estimating total crossing time.

\subsection{Analysis influence of pedestrian demand on pedestrian platoon dispersion.}

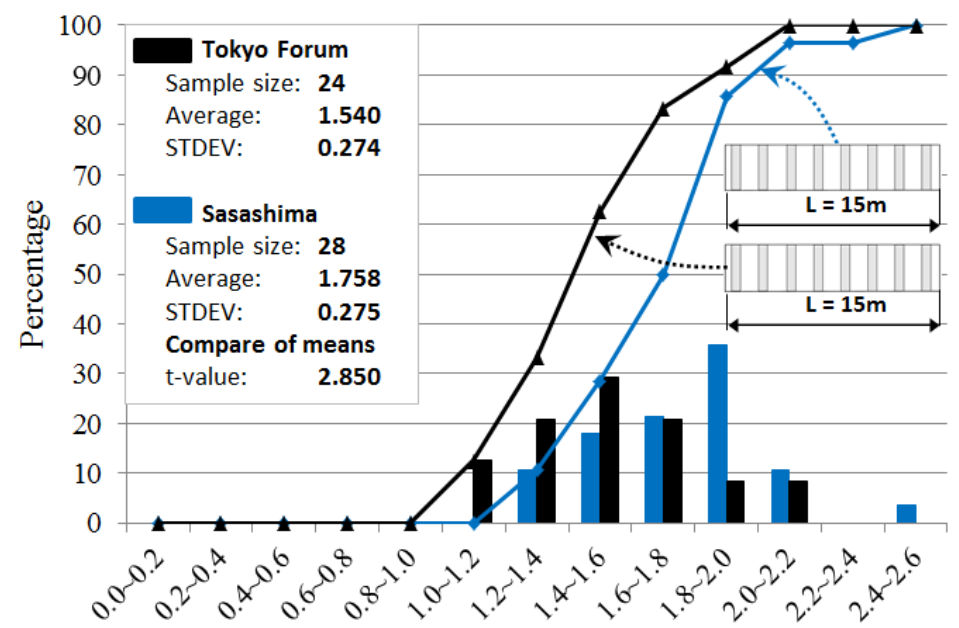

Platoon dispersion ratio

Figure 7. PDR at Tokyo forum and Sasashima crosswalk. 
Transport and Communications Science Journal, Vol. 71, Issue 1 (01/2020), 1-10

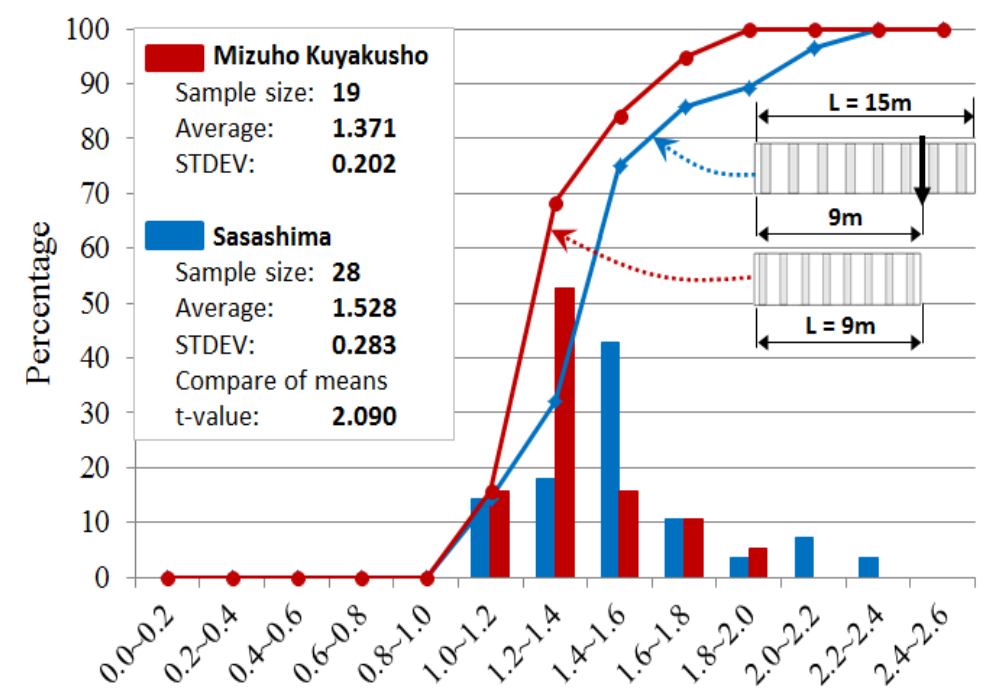

Platoon dispersion ratio

Figure 8. PDR at Mizuho Kuyakusho and Sasashima crosswalk (at 9 meters length).

Before analyzing the influence of pedestrian demand, it is important to note that Sasashima crosswalk has a demand three times higher than Mizuho Kuyakusho and Tokyo forum crosswalk. Fig. 7 shows the distribution of PDR at Tokyo forum and Sasashima crosswalk. From Fig. 7, as expected, the pedestrian demand has a significant influence on pedestrian platoon dispersion. The distribution of PDR at Sasashima crosswalk is higher than that at Tokyo crosswalk although both crosswalks have the same crosswalk length. Fig. 8 presents the distribution of PDR at Mizuho Kuyakusho and a reference line of 9 meters from the upstream curb at Sasashima crosswalk. The result supports the fact that that with the same length, the crosswalk with higher demand has greater PDR.

\subsection{Analysis influence of crosswalk length on pedestrian platoon dispersion.}

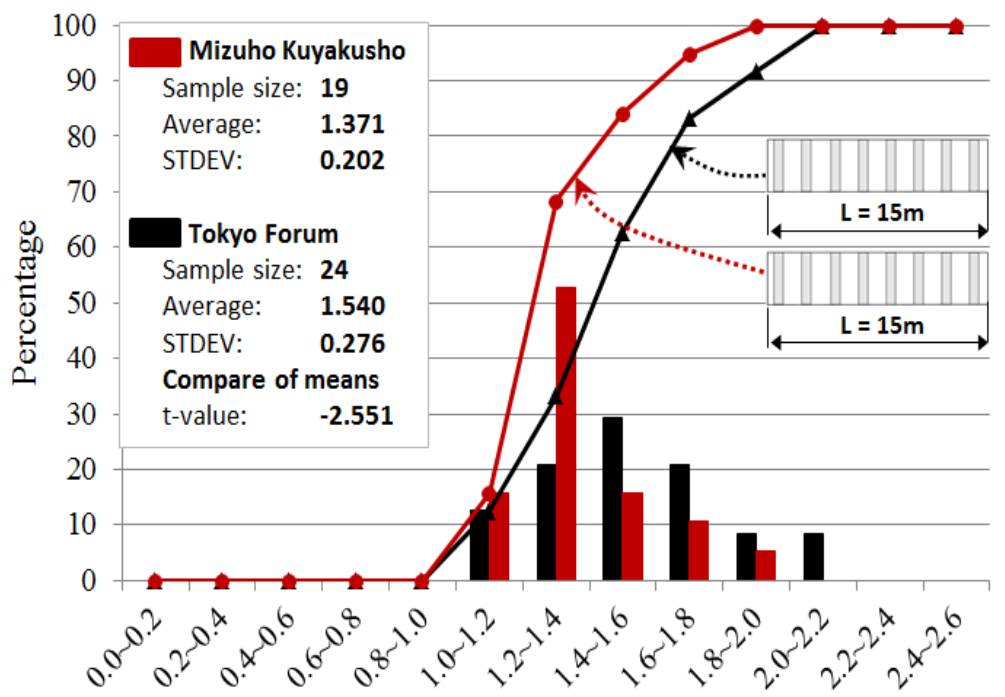

Platoon dispersion ratio

Figure 9. PDR at Mizuho Kuyakusho and Tokyo forum crosswalk. 
Transport and Communications Science Journal, Vol. 71, Issue 1 (01/2020), 1-10

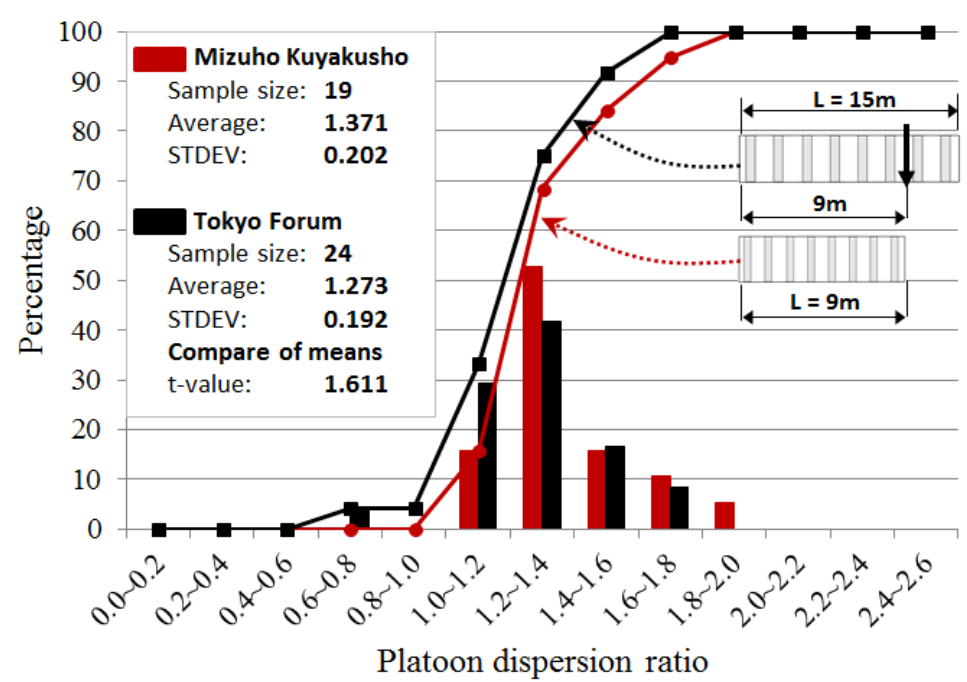

Figure 10. PDR at Mizuho Kuyakusho and Tokyo forum crosswalk (at 9 meters length).

Fig. 9 shows the platoon ratio at Mizuho Kuyakusho crosswalk and Tokyo forum intersection. It should be emphasized here that these crosswalks have similar pedestrian demand. It is clear that the dispersion ratio at Tokyo crosswalk with 15-meter length is higher than that at Mizuho Kuyakusho crosswalk with 9 meters length. In addition, PDR is also estimated at a reference line of 9 meters from the upstream curb at Tokyo crosswalk and is presented in Fig. 10. It is interesting to say that, at the same length, PDRs of these two crosswalks are not significant different although the dispersion at Mizuho Kuyakusho is little bit higher compared with that at Tokyo crosswalk with 9-meter length. This can be interpreted as the utility of the lower pedestrian demand at Tokyo crosswalk compared to Mizuho Kuyakusho crosswalk demand. The influence of crosswalk length upon platoon dispersion can be explained as the longer crosswalk is, the more crossing time is. Therefore, pedestrians will have more opportunities to deviate from average crossing time.

\section{CONCLUSION AND FUTURE WORK}

Existing methodologies for estimating pedestrian crossing time requirement do not consider the effects of platoon dispersion. The dispersion phenomenon should be taken satisfactorily to estimate the crossing time requirement because if a great dispersion happens, pedestrians might not have sufficient time to complete their crossing.

In this paper, a pedestrian platoon dispersion index (PDR) was proposed to analyze the influences of pedestrian demand and crosswalk length. Data at three signalized crosswalks were collected and processed for the estimation the PDR. It is concluded that, the higher demand is, the greater dispersion occurs. Regarding crosswalk length, platoon dispersion becomes greater as crosswalk length increases.

In this paper, the analysis is limited to only two factors: pedestrian demand and crosswalk length. Since pedestrian platoon dispersion may be influenced by other factors such as bi-directional flow, crosswalk width, pedestrian origin-destination, pedestrian type, etc., more data need to be collected in the future to identify the other influencing factors. Moreover, model will be developed to quantify the dispersion of pedestrian platoon under different demand levels, crosswalk geometries and operational policies. 


\section{REFERENCES}

[1] Traffic Safety Basic Facts - Pedestrians. European Road Safety Observatory, 2018

[2] Traffic Bureau, Japan National Police Agency: Statistics of Traffic Accidents, 2019.

[3] Highway Capacity Manual (HCM), National Research Council, Washington, D.C., USA, 2010

[4] Japan Society of Traffic Engineers, Manual of Traffic Signal Control. Tokyo, Japan, 2006.

[5] V. Thamizh Arasan, Shiraj Hussain Kashani, Modeling Platoon Dispersal Pattern of Heterogeneous Road Traffic, CD-ROM, Presented at TRB 2003 Annual Meeting

[6] M. R. Virkler, Signal Coordination benefits for pedestrians,Transportation Research Record, 1636 (1998) 77-82. https://doi.org/10.3141/1636-12

[7] M. R. Virkler, Scramble and Crosswalk Signal Timing, Transportation Research Record, 1636 (1998) 83-87. https://doi.org/10.3141/1636-13

[8] Japan Society of Traffic Engineers, Manual of Traffic Signal Control. Tokyo, Japan, 2006.

[9] A. Forde, J. Daniel, Performance evaluation of the HCM 2010 platoon dispersion model under midblock pedestrian and truck traffic friction conditions, Journal of traffic and transportation engineering (English edition), 4 (2017), 522-534. https://doi.org/10.1016/j.jtte.2017.04.003

[10] K. Suzuki, H. Nakamura, TrafficAnalyzer - The Integrated Video Image Processing System for Traffic Flow Analysis, CD-ROM. Presented at 13th World Congress on Intelligent Transportation Systems, 8 pages, London, 2006.

[11] W.K. Alhajyaseen, M. Li, H. Nakamura, W. Daamen, Effectiveness of Signal Coordination for Pedestrian Flows Considering Bi-directional Flow Impacts, Asian Transport Studies, 2 (2013) 223238. https://doi.org/10.11175/eastsats.2.223 